\title{
Bildungssprache in Elternhaus und Schule
}

\author{
Wolfgang Steinig
}

Online publiziert: 27. Januar 2020

(C) Der/die Autor(en) 2020

Zusammenfassung In sozial differenzierten Familienwelten herrschen charakteristische Erziehungsmethoden, in denen Kinder sprachlich differente Gesprächsmodi entwickeln. Gegenüber familialer Kommunikation ist die Kommunikation im Unterricht deutlich rigider und bietet für Kinder aus ressourcenarmen und bildungsfernen Familien wenig Möglichkeiten, eine bildungssprachlich angemessene Ebene zu finden, um fachliche Gespräche zu führen. Um die Defizite unterrichtlicher Kommunikation zu mindern und die Chancen zu erhöhen, mangelnde bildungssprachliche Kompetenzen zu kompensieren, werden didaktische Vorschläge gemacht.

Schlüsselwörter Bildungssprache $\cdot$ Erziehungsmethoden · Familienwelten ·

Gesprächskultur · Klassifikation · Kulturelles Kapital · Rahmung · Soziale Schicht · Soziale Ungleichheit · Unterricht

\section{Development of Educational Language at Home and at School}

\begin{abstract}
In families from different social strata characteristic educational methods prevail, in which children develop different linguistic modes of expression. When compared to communication within the family, communication in the classroom is much more rigid and offers little opportunity for children from low-resource and educationally disadvantaged families to find an adequate level of education-related language with which to conduct professional discussions. In order to reduce the deficits of lesson communication and increase the chances of compensating for lack of educational language competencies, didactic suggestions are made.
\end{abstract}

\footnotetext{
W. Steinig $(\bowtie)$

Philosophische Fakultät, Germanistisches Seminar, Universität Siegen, Siegen, Deutschland

E-Mail: steinig@germanistik.uni-siegen.de
} 
Keywords Language of Education · Educational Methods · Family Worlds · Conversation Culture · Classification · Cultural Capital · Framing · Social Class · Social Inequality · Teaching

\section{Einführende Bemerkungen}

Bildung und Sprache, mit der Bildung kommuniziert wird, bilden eine Einheit. Aber in pädagogischer, psychologischer und soziologischer Literatur findet man eine breite Forschungstradition, in der bildungssprachliches Verhalten zwar immer mal wieder kurz erwähnt, aber nicht substantiell eingebunden wird. Dabei wäre es dringend erforderlich, in den soziologischen Konstruktionen zur Ungleichheit das sozial differente Sprachverhalten als einen konstitutiven Bestandteil zu integrieren. Dazu soll dieser Aufsatz einen Beitrag leisten.

Exemplarisch für die soziologische Forschungstradition zur Bildung kann Pierre Bourdieu (1987) stehen, der mit dem sozialen Konstrukt >Habitus< erkenntnisreiche Analysen zu Einstellungen und zum Verhalten in unterschiedlichen sozialen Milieus ermöglichte, der aber die sprachliche Varianz, die mit der habituellen Varianz einhergeht, nicht näher untersucht hat. In dem 2011 von Andreas Lange und Margret Xyländer herausgegebenen Sammelband >Bildungswelt Familie<wird auf eine Vielzahl an Diskursen zur Bildung verwiesen, die sich letztlich alle mehr oder weniger auf das Bourdieu'sche Habitus-Konstrukt zurückführen lassen mit seinen engen Bezügen zum sozialen Status und zur Bildung als habitueller Attitüde sowie ihren zeitgebundenen politischen und ökonomischen Bedingungen: einer verschärften Konkurrenz in einer globalisierten Welt, dem Übergang von der Dienstleistungszur Wissensgesellschaft, veränderten Erziehungspraktiken in Familien und schulisch induzierte Selektionsmechanismen, um nur einige Facetten dieser vielschichtigen Diskussion zu nennen.

Neben diesen öffentlichen Diskursen hat sich die Forschung zu sozial bedingter Bildungsungleichheit nach den enttäuschenden Ergebnissen der internationalen Schulleistungstests von PISA und IGLU intensiviert. Während in den 1960er/70er Jahren der Fokus der Ungleichheitsforschung mit Begriffen wie >Sprachbarrieren und der >Code-Theorie< Basil Bernsteins (1971) noch soziolinguistisch geprägt war, spielt heute die Soziolinguistik nur noch eine marginale Rolle. Stattdessen möchte man das familiale Bildungsgeschehen ethnographisch und mikrosoziologisch durchleuchten, um seine Prozesse und Wirkungen besser verstehen zu können, denn in der familialen Sozialisation sieht man, damals wie heute, das entscheidende Agens, wie sich Bildung in der Kindheit entwickelt und von Generation zu Generation tradiert wird, während schulische Bildung das familiale Bildungskapital nicht grundlegend verändert. Kinder in bildungsnahen Familien bleiben in ihrem Milieu, während Kinder aus bildungsfernen Familien trotz schulischer Bildung selten in bildungsaffine Milieus aufsteigen. Das gilt in besonderer Weise für Deutschland, wo der schulische Erfolg stärker von der sozialen Herkunft abhängt als in kaum einem anderen Land. 


\section{Sprachlich differente Entwicklungen in der Familie}

Sprache entwickelt sich bei jedem Kind normalerweise in einer überaus zuverlässigen, robusten Weise. Dennoch zeigen sich bereits früh quantitative wie qualitative Unterschiede, je nachdem in welchem familialen Milieu ein Kind aufwächst. Während einerseits manche Eltern viel und auf anspruchsvollem Niveau mit ihren Kindern sprechen, ist man in anderen Familien schweigsamer und legt auf einen differenzierten Ausdruck weniger Wert. Betty Hart und Todd Risley haben 1995 in den USA eine bis heute wegweisende Langzeitstudie zur Eltern-Kind-Kommunikation vorgelegt. Sieben Monate alte Kinder aus 42 Familien wurden über zweieinhalb Jahre jeweils eine Stunde im Monat bis zum Alter von drei Jahren videographiert und ihr Sprachverhalten analysiert: die Äußerungen der Kinder und die Äußerungen, die an sie gerichtet wurden, sowie all das, was sonst noch während der Aufnahmezeiten gesprochen wurde. So ergab die quantitative Analyse des Wortumfangs von Äußerungen, die arbeitslose Eltern, die auf Sozialhilfe angewiesen sind (on welfare), in einer Stunde an ihre Kinder richteten, durchschnittlich 616 Wörter. Eltern aus dem Arbeitermilieu (working class) äußerten 1.251 Wörter stündlich, also etwa doppelt so viele, und Eltern aus der oberen Mittelschicht (professionals) kamen auf 2.153 Wörter.

Wie lassen sich diese enormen Unterschiede erklären? Die Menge der Äußerungen, die sich auf die Organisation des familialen Alltags beziehen, war in allen sozialen Schichten ungefähr gleich. Doch in Familien aus der Arbeiterschicht und noch sehr viel mehr in Familien der oberen Mittelschicht sprachen die Eltern mit ihren Kindern darüber hinaus über weit mehr: über Vergangenes und Zukünftiges, über selbst Erlebtes oder von anderen Personen oder aus Medien Erfahrenes, über Einschätzungen eigener oder fremder Verhaltensweisen, kurz, über alles Mögliche, das nicht im Hier und Jetzt des familialen Alltags anstand, sondern über diesen Kontext hinausging: Vergangenes und Zukünftiges sowie imaginierte Vorstellungen.

Die Aufgabe einer ethnographisch-soziolinguistisch profilierten Forschung bestünde darin, in die unterschiedlichen Familienwelten hineinzuschauen und hineinzuhorchen, um zu entdecken, wie sich kindliches Wissen und Bildung in der Kommunikation mit den Eltern, im Umgang mit Medien, in der Peer Group und schließlich in der Schule entwickelt. Ausgehen sollte man bei diesem Forschungsprogramm bei den Mutter-Kind-Dyaden unmittelbar nach der Geburt. Bereits hier wird deutlich, dass einige Mütter viel sprechen, während andere eher schweigsam sind. Aber eine >beredte< Mutter wird gegenüber einer >schweigsamen $<$ Mutter bereits in diesem frühen Alter ein grundsätzlich anderes Verhältnis im Umgang mit Sprache etablieren, denn das von Eltern an ihr Kind gerichtete Sprechen, das weit über das Minimum hinausgeht, um den Alltag im Hier und Jetzt zu bewältigen, erzeugt früh ein angenehmes Gefühl liebevoller Nähe und regt zur Nachahmung an.

Die ersten bildungssprachlich relevanten Äußerungen von Eltern lassen sich bereits im Kleinkindalter beobachten. Eltern aus bildungsnahen Milieus richten häufig Äußerungen in lustvoller Weise auf relativ hohem Niveau an ihre kleinen Kinder, ohne erwarten zu können, dass ihre Äußerungen verstanden werden. Vorsprachliche Lautmuster und normabweichende kindliche Äußerungen werden von gebildeten Eltern oft lustvoll imitiert, ohne ängstlich zu sein, dass sich diese Muster verfestigen 
könnten. Durch das ständige sprachliche Miteinander, unabhängig davon, ob dadurch etwas semantisch Dekodierbares vermittelt wird oder nicht, entsteht offensichtlich ein korrespondierendes sprachliches Band zwischen Eltern und Kindern, das zur gegenseitigen Nachahmung und das Kind zum Imitationslernen anregt (Hart/Risley 2007, S. 199). Sprachliche Imitation ist bei Kindern ohnehin ein zentrales Agens des Spracherwerbs, aber wenn die Aufmerksamkeit auf sprachliche Äußerungen durch eine sprachlich reiche Eltern-Kind-Resonanz zusätzlich befeuert wird, entsteht ein sprachlicher Mehrwert im Lerngeschehen.

Erste bildungssprachliche Spuren kann man bereits bei dreijährigen Kindern beobachten. Dazu eine mir berichtete Begebenheit. Ein Junge, nicht älter als drei Jahre, sitzt früh am Morgen alleine im Schlafanzug vor dem Hund einer Familie, bei der er mit seinem Vater zu Gast ist. Sein Vater kommt nach einer Weile leicht beunruhigt hinzu und fragt den Kleinen, ob alles in Ordnung sei und ob er nicht lieber zurück ins Bett möchte. Darauf erwidert er: Papa, ich komm schon allein zurecht. Damit wollte er sagen, dass der Hund ihm keine Angst macht und er Herr der Lage sei. Diese Äußerung erstaunte nicht nur seinen Vater, sondern alle Erwachsenen, denen diese Begebenheit später berichtet wurde. Man hatte diese Formulierung von einem drei Jahre alten Kind, das noch Windeln benötigt, nicht erwartet. Mehrfach wurde seine Äußerung später in Gesprächen im erweiterten Familienkreis kolportiert und man war einhellig der Meinung, dass dieses Kind außergewöhnlich begabt sei und später in Schule und Beruf Erfolg haben würde. Die Aufmerksamkeit und Wertschätzung, die solch ein Kind nicht nur durch diese Äußerung von seinen Eltern erfährt, wird dazu beitragen, dass es sie verstärkt zu beachten und zu adaptierten versucht. Mit besonderen Formulierungen bei Zuhörern zu punkten kann so zur Basis einer rhetorischen Kompetenz werden, einer Kompetenz, die stärker auf den aktiven, performativen Aspekt sprachlichen Handelns zielt als der Begriff >Bildungssprache<, dem immer etwas Statisches, Rezeptives und Schriftsprachliches anhaftet.

Wie lassen sich empirisch in Familien bildungssprachlich relevante Situationen erfassen, ohne sich auf zufällig erfahrene Anekdoten berufen zu müssen? Zum einen kann man so vorgehen, wie in der bereits erwähnten Studie von Hart und Risley (1995), nämlich über einen längeren Zeitraum systematisch zu videographieren, was sich in zufällig ausgewählten Zeitspannen in einer Familie sprachlich ereignet. Bei diesem quantitativ orientierten Verfahren benötigt man allerdings sehr viele Sprachaufnahmen, um das durchschnittliche und charakteristische sprachliche Geschehen in einer Familie zu erfassen. Geht man hingegen qualitativ vor, sollte man sich auf bestimmte Zeiten im Familienalltag konzentrieren, in denen zu erwarten ist, dass sie bildungssprachlich dichter sind.

Margret Xyländer (2014) hat sich in ihrer Studie zur »Familie als Bildungsgemeinschaft « auf Abendrituale fokussiert. Sie geht darin von der Vorstellung eines Doing Family aus und meint damit Prozesse und Praktiken, mit denen sich eine Familie mehr oder weniger bewusst darauf einrichtet, zu bestimmten Zeiten in besonderer Weise als Familie zu agieren. Diese Zeiten haben einen rituellen Charakter, da sie nach einem mehr oder weniger festgelegten Muster ablaufen, beispielsweise während eines gemeinsamen Abendessens am Samstagabend, an dem die ganze Familie auf bestimmten Plätzen an einem gut gedeckten Tisch sitzt, manchmal gerahmt durch bestimmte Einleitungsformeln, Lieder, Texte oder Gebete. In diesen 
familial dichten Situationen wird in vielfältiger Weise miteinander gesprochen; man zeigt, dass man in einer für alle wertvollen Zeit aufeinander eingeht und miteinander über mehr und anderes und in einer anderen Weise spricht, als im normalen Alltagsgeschehen.

Charakteristisch für diese rituellen Situationen sind Erzählungen zu unterschiedlichsten Beobachtungen und Begebenheiten und ihre Einordnung und Bewertung, an der sich Erwachsene wie Kinder beteiligen. Hier wird kulturelles Familienkapital prozessiert, an dem Kinder partizipieren können und so eine familial geprägte Bildung aufbauen, die ihnen für ihr gesamtes Leben, nicht nur für die Schule, mehr oder weniger nützlich sein kann. Die Familie wird besonders in diesen rituellen Situationen zur primären und prägenden Bildungsinstanz. Die kognitiven und sprachlichen Bildungsprozesse, die sich hier entwickeln, lassen sich nicht auf einen sprachlichen Bildungswortschatz reduzieren, der zwar nebenher auch erworben wird. Entscheidend ist vielmehr, wie mit Wissen kommunikativ im Familienkreis umgegangen wird. Wer wirft eine Frage auf? Wer geht darauf ein? Wie wird erzählt, wie wird argumentiert und wie wird etwas erklärt? Die bildungssprachliche Lexik und linguistische Merkmale, die Bildungssprache angeblich konstituieren, spielen hier eine untergeordnete Rolle. Entscheidend sind vielmehr die sprachlichen Handlungen und die rhetorischen Verfahren, mit denen man sich aktiv an Gesprächen beteiligt (vgl. Steinhoff 2019).

Dass sich eine Familie als Bildungsgemeinschaft begreift, ist allerdings ein Selbstverständnis, dass nicht in allen sozialen Milieus beobachtet werden kann. Wenn man von den SINUS-Milieus ausgeht, einem soziologischen Modell, das neben der ökonomischen Basis auch die Lebenswelten und Einstellungen von Familien einbezieht ${ }^{1}$, wird man erkennen, wie unterschiedlich Bildung kommunikativ in den familialen Alltag integriert wird. In ökonomisch und sozial-kulturell ressourcenärmeren Milieus wird man in geringerem Umfang und kommunikativ voraussagbarer bildungsaffine Bereiche berühren. Doing Family in sprachlich dichten rituellen Situationen wird man hier kaum betreiben. In ökonomisch gut situierten Familien, die im SINUSModell für Beschleunigung, Multioptionalität, Exploration und neue Synthesen stehen, wie etwa das expeditive Milieu, wird man vermutlich ebenfalls beobachten können, dass bildungsbürgerliche Vorstellungen eines Doing Familiy mit ElternKind-Gesprächen bei gemeinsamen Mahlzeiten kaum noch gepflegt werden, da individuell gegessen wird und die Rezeption von Inhalten aus dem Internet ElternKind-Gespräche einschränkt. Doch systematisch angelegte milieuspezifische Studien zur Kommunikation in Familien gibt es noch nicht, wären aber notwendig, da mit der herkömmlichen Mittelschicht-Unterschicht-Dichotomie die zunehmend differenzierter werdenden Familienwelten nicht erfasst werden können. Hinzu kommen durch Migration und Mehrsprachigkeit bedingte Einflüsse, die bildungssprachliches Verhalten modifizieren.

\footnotetext{
1 https://www.sinus-institut.de/fileadmin/user_data/sinus-institut/Bilder/Sinus-Milieus_092018/201809-18_Informationen_zu_den_Sinus-Milieus.pdf] (gesehen am 28.11.2019).
} 
Bei Tischgesprächen in der Familie mit Kindern im Alter von circa 6 Jahren konnten Miriam Morek (2012) und Vivien Heller (2012) drei unterschiedliche Interaktionsmuster beim Erklären und Argumentieren identifizieren:

1. Fordern und Unterstützen,

2. Übergehen und Belehren,

3. Dulden und Fallenlassen.

Eltern, die beim Tischgespräch ihre Kinder fordern und unterstützen, betreiben ein aktives Scaffolding. Sie unterbrechen nicht und geben ihnen ausreichend Zeit, ihre Äußerungen abzuschließen. Nachfragen führen dazu, dass die Kinder ihre Äußerungen umformulieren, erweitern und präzisieren. Kinder können so ihre Sprachkompetenz nicht nur beim Erklären und Argumentieren entwickeln, sondern ein dekontextualisiertes, literales Ausdrucksvermögen erwerben, das für den schulbezogenen Sprachgebrauch in konzeptionell mündlicher wie schriftlicher Modalität vorteilhaft ist.

Andere Eltern können leicht ungeduldig werden, wenn ihre Kinder zu langsam, ungenau oder zu umständlich formulieren: Sie unterbrechen sie, entziehen ihnen das Wort und formulieren selbst, was das Kind ihrer Meinung nach sagen wollte. Die kindliche Äußerung wird nicht ernst genommen und ignoriert, da die Eltern den Sachverhalt, das Argument oder die Erklärung angemessener ausführen können, oft in einem belehrenden Ton. Falls die Kinder versuchen sollten, die Äußerungen der Eltern zu diskutieren, werden sie übergangen. Die Eltern agieren als Erwachsene, die alles grundsätzlich besser wissen als Kinder und keine Einsprüche dulden - eine Attitüde, die vielfach auch Lehrkräfte einnehmen. Kinder eignen sich so zwar durch die Belehrungen der Eltern, ähnlich wie beim Lehrervortrag, deklaratives Wissen und einen rezeptiven Bildungswortschatz an, aber da sie sich nicht aktiv am Gespräch beteiligen können, wird ihre kommunikative Kompetenz nur wenig gefördert.

Das dritte Interaktionsmuster >Dulden und Fallenlassen $<$ herrscht in Familien mit konservativen Rollenvorstellungen vor. Hier wird Kindern grundsätzlich ein Mitspracherecht verweigert, wenn sich Erwachsene unterhalten. Sollten sie dennoch versuchen, sich am Gespräch zu beteiligen, werden ihre Äußerungen allenfalls geduldet, aber man geht nicht darauf ein: Sie werden übergangen.

Neben den Gesprächen am Mittags- oder Abendtisch ist das Vorlesen aus Bilderbüchern ein zentrales Element familialer Rituale. Für die Entwicklung von Literacy und Bildungssprache hat die Vorlesesituation eine nicht zu unterschätzende Bedeutung. Man kann davon ausgehen, dass die weitaus meisten Eltern aus allen sozialen Milieus um die Bedeutung des Vorlesens für die Entwicklung von Sprache und Lesemotivation wissen. Den meisten Eltern wird aber kaum bewusst sein, dass es zwar wichtig ist, vorzulesen, aber dass darüber hinaus die Art und Weise wie das geschieht, wohl noch bedeutsamer ist. Um zu erkennen, wo die Unterschiede zwischen einem für die Entwicklung von Sprache und Kognition förderlichen und einem weniger sinnvollen Vorlesen liegen, muss man zunächst beobachten, wie Eltern und Kinder in Vorlesesituation sprachlich agieren.

Williams (2001) hat dazu Mütter mit ihren Kindern aus unterschiedlichen sozialen Milieus in der Vorlesesituation videographiert und dabei bedeutsame Unterschiede feststellen können. Die australischen Familien, aus denen die Mütter stammten 
(unterschiedliche Stadtviertel in Melbourne), unterschieden sich hinsichtlich der relativen Autonomie des (männlichen) Ernährers am Arbeitsplatz, der über mehr oder weniger große Entscheidungsbefugnisse verfügt. Sie unterschied in ihrer Studie dementsprechend zwischen Familien mit niedriger und hoher Autonomie. Dahinter steht die marxistische Annahme von Basil Bernstein (1971), dass die Arbeit als materielle >Basis< des Seins den >Überbau< mit seinen kulturell differenten Ausprägungen habitueller Muster konfiguriert, die die (sprachliche) Erziehung in Familienwelten prägen.

In den Vorlesesituationen ließ sich u.a. beobachten, dass Mütter aus Familien mit niedriger Autonomie sich nahezu ausschließlich auf das im Buch Sichtbare beziehen, dazu Fragen an das Kind richten und Informationen geben. Die Fragen der Kinder werden häufiger übergangen, vor allem dann, wenn sie über das Gesehene hinausgehen. Diese Eltern unterstützen höchst selten einen Transfer zwischen dem momentan gelesenen Text mit seinen dazu gehörenden Bildern und den Gedanken, Erfahrungen und Beobachtungen, die außerhalb des Bilderbuches liegen, sei es in der gemeinsamen Lebenswelt, in fiktionalen Welten oder in sprachlichen Bezügen.

Mütter aus Familien mit hoher Autonomie geben dagegen meist nicht einfach nur eine kurze Antwort auf Fragen des Kindes, sondern beziehen in ihren Kommentaren auch Lebensbereiche ein, die über das im Buch Gestaltete hinausgehen. Als beispielsweise ein etwa vierjähriges Kind im Bilderbuch einen Wolf sieht, der sich mit einem Schafspelz verkleidet hat und seine Mutter fragt »What is it? « antwortet sie: »He's pretending to be a sheep. That's what you call a wolf in sheep's clothing. « (Williams 2001, S. 28).

Die Mutter gibt ihrer Tochter eine semantische Information zu einer Redensart, deren weitreichende idiomatische Implikationen ihr Kind in diesem Alter zwar noch nicht versteht, ihm aber bereits einen ersten Hinweis gibt, in welcher Richtung die Metapher >Wolf im Schafspelz< unabhängig von der konkreten Visualisierung im Kinderbuch als kognitiver Frame verstanden wird, andere über seine wahre Identität zu täuschen. Wenn man mit Lakoff (1987) davon ausgeht, dass unsere Kognition durch idealisierte kognitive Modelle organisiert wird, die sich in Metaphern manifestieren, dann ermöglicht diese Mutter ihrer Tochter beim gemeinsamen Lesen eines Bilderbuchs diese Metapher für ein generelles Täuschungsverfahren als Frame in einem ersten vorläufigen Schritt zu erfassen. Ein möglichst dichtes Netz neuronaler Verknüpfungen aufzubauen, gelingt nur, wenn Wörter oder Wortgruppen mit sensualen Eindrücken und metaphorischen Mustern verbunden werden können. Mit dem Hinweis der Mutter auf eine bildliche Redensart wird ein kognitives Fenster geöffnet, dass dem Kind später einmal grundsätzlich ermöglichen wird, das Funktionieren metaphorischer Ausdrücke zu erkennen und im Rahmen der Entwicklung eines literalen Sprachgebrauchs zu nutzen.

Nicht nur das interaktive Vorlesen aus Bilderbüchern (Steinig/Huneke 2015, S. 209 f.), auch das gemeinsame Anschauen einer Fernsehsendung kann für eine elaborierte Sprachentwicklung nützlich sein, nämlich dann, wenn sie von Eltern wie Kindern immer wieder kommentiert wird. So kann ein banales Trash-Format wie etwa »Deutschland sucht den Superstar« dazu dienen, kommentierende Sprachhandlungen angemessen zu formulieren, in höherem Alter dann auch mit witzigen und ironischen Elementen versetzt, die einem jugendsprachlich-respektlosen oder bil- 
dungsbürgerlich-distanzierenden Habitus entsprechen können. In vielen Familien wird zwar auch gemeinsam Fernsehen geschaut, aber hier dann schweigend und unkritisch rezipierend. Kritische oder ironische Kommentare sind nicht erwünscht, erst recht nicht von Kindern; sie würden das stumme Genießen stören.

Dichte familiale Situationen entstehen auch in den Ferien, besonders auf der gemeinsamen Reise im Auto. Hier können Kinder von der Kommunikation mit den Eltern ausgeschlossen bleiben oder aber in Gespräche verwickelt werden, die dicht und ertragreich sein können. Die Ferienzeit ist für die Forschung interessant, da man hier den Einfluss der Familie auf die literale Entwicklung von Kindern unabhängig von schulischen Einflüssen beobachten kann. In Studien zum sog. >Summer-Learning-Loss $<$ in den USA, wo die Sommerferien besonders lang sind, wurde deutlich, dass Kinder aus Familien mit niedrigem sozialem Status besonders in ihren literalen Kompetenzen um etwa ein bis zwei Monate bis zum Wiederbeginn der Schulzeit zurückfallen, während Kinder aus der Mittelschicht ihren Entwicklungsstand halten oder sogar noch weiter ausbauen konnten (Entwisle/Alexander/Olson 2000).

Literacy-Programme, mit denen versucht wird, die literalen Defizite von Kindern aus unterprivilegierten Schichten zu kompensieren, scheinen nur dann erfolgreich zu sein, wenn man die Eltern der Kinder mit einbezieht (Graham/McNamara/Van Lankveld 2011). Aber diese Interventionsprogramme zeigen meist keine nachhaltige Wirkung, da man die Lebenswelt von Familien, in der es nur geringe oder keine Affinitäten zur Bildung und bildungssprachlichem Verhalten gibt, nicht nachhaltig verändern kann.

Welche der möglichen interaktiv dichten familialen Situationen man auch betrachtet - die Gespräche beim Essen, beim Vorlesen, beim Fernsehen oder in den Ferien -, immer schält sich ein bestimmtes Muster heraus, das in einem mehr oder weniger direkten Bezug zu den sozial differenten Familienwelten steht: in ressourcenreichen Familien mit einem ökonomisch und kulturell höherem Kapital kommunizieren Eltern nicht nur häufiger mit ihren Kindern, sondern auch anspruchsvoller, vielschichtiger, variantenreicher, konzeptionell schriftlicher, dekontextualisierter, kognitiv fordernder und bildungsaffiner als in ressourcenärmeren Familien.

Affinitäten zwischen sozialen Milieus, ihren Erziehungsvorstellungen und ihrem Sprachverhalten lassen sich nicht als statische Äquivalenzen festschreiben; sie unterliegen einem stetigen sozialen Wandel. Nach Doepke/Zilibotti (2019) hängen Erziehungsmethoden in hohem Maße davon ab, in welchem Ausmaß sie den ökonomischen Erfolg der nachwachsenden Generation beeinflussen. Investitionen in Erziehung lohnen sich vor allem in Ländern mit einer ausgeprägten sozialen Ungleichheit. In Ländern mit geringer Ungleichheit werden mit erzieherischen Investitionen dagegen geringere Renditen erzielt, etwa in den skandinavischen Ländern. Hier pflegen die Eltern eher einen entspannten, permissiven Erziehungsstil; ihr Druck auf Kinder, sich in der Schule anzustrengen, um gute Noten und Abschlüsse zu erreichen, ist deutlich geringer als in Ländern mit hoher sozialer Ungleichheit, vor allem in Ländern wie China, den USA oder Großbritannien. Aber seit den 1980er Jahren sind auch in Deutschland die ökonomischen und sozialen Disparitäten enorm angewachsen. Das hat dazu geführt, dass vor allem Eltern aus der sozialen Mittelschicht von einem permissiven zu einem autoritativen Erziehungsstil übergegangen sind, der durch ein höheres Maß an Steuerung und Kontrolle gekennzeichnet ist. Autoritative 
Erziehung wird nicht, wie die autoritäre Erziehung, durch Befehle und Disziplin geprägt, sondern durch eine mehr oder weniger geschickte Einflussnahme auf Einstellungen und Werte des Kindes, die zu einer Selbststeuerung und Selbstdisziplin mit hohen Leistungserwartungen führen soll (Steinig 2017: $163 \mathrm{ff}$., Weininger/Lareau 2009). Wenn zum autoritativen Erziehungsstil noch autoritäre Praktiken hinzukommen, nennen das Doepke/Zilibotti (2019) >intensive parenting <, ein Erziehungsstil, den sie den sog. Helikopter-Eltern zurechnen.

Das Phänomen der Helikopter-Eltern lässt sich aus dem Bemühen heraus erklären, in einer zunehmend an Konkurrenz orientierten Gesellschaft mit geringeren Aufstiegschancen und Ängsten vor sozialem Abstieg die eigenen Kinder so intensiv wie möglich zu fördern, um in diesem schärfer werdenden Wettbewerb um privilegierte Positionen bestehen zu können. Bereits im Vorschulalter bekommen Kinder innerhalb und außerhalb der Familie Bildungsangebote. Das setzt sich fort in der Grundschule mit Nachhilfestunden, bereits dann, wenn die Leistung nur leicht zurückgeht, meist auch von der Sorge getrieben, der Übergang zum Gymnasium könnte nicht gelingen; auch die Bemühungen, möglichst gute (Privat)schulen und Universitäten zu finden oder hochwertige Praktika und Auslandsaufenthalte zu ermöglichen, gehören in diesen Kontext. Helikopter-Eltern versuchen, das Leben ihrer Kinder mit möglichst viel kulturellem Kapital auszustatten und Einflüsse, die dieses Ziel beeinträchtigen könnten, zu minimieren.

Während Eltern mit einem permissiven Erziehungsstil die Bedürfnisse eines Kindes im Hier und Jetzt nach Autonomie und freiem Spiel befriedigen möchten und sich nicht zu viel um seine Zukunft sorgen, haben Helikopter-Eltern die Zukunft ihrer Kinder im Blick. Sie glauben zu wissen, was ihnen im Leben nützen wird, nämlich schulisch relevantes Wissen, aber auch kulturelles Wissen, das über das schulische Curriculum hinausgeht (Doepke/Zilibotti 2019, S. 36 ff.). In Gesprächen beim Essen wird es in Familien mit diesen Eltern daher häufiger um schulisch relevante Themen gehen, etwa um Strategien, wie man die Leistungsbilanz in der Schule, aber auch im Tennisverein oder Schachclub verbessern kann.

Studien zeigen, dass Kinder, die autoritativ erzogen werden, vergleichsweise bessere Schulleistungen aufweisen als permissiv erzogene (Doepke/Zilibotti 2019, S. 33; Xyländer 2014, S. 35). Wie stark sich die Effekte der seit den 1970er Jahren intensivierten Erziehungspraxis auf die Steigerung schulisch relevanter Kompetenzen auswirken, zeigen Untersuchungen zur Erklärung von PISA-Ergebnissen: Wenn Eltern aus Büchern vorlesen, Geschichten erzählen und zudem noch über Politik diskutieren, liegt das PISA-Ergebnis um 32 Punkte über dem von Kindern, in denen keine dieser Aktivitäten vorkommt: »... an intensive parenting style is associated with a significant improvement in school performance.« (Doepke/Zilibotti 2019, S. 76).

Während man in Mittelschichtfamilien einen Wandel vom permissiven zum autoritativen Erziehungsstil beobachten kann, sind in Familien aus der Unterschicht zwei Stile vorherrschend: ein autoritärer und einer, den Maccoby/Martin (1983, S. 39) als »neglecting, ignoring, indifferent, univolved « (vernachlässigend) bezeichnen. Zum Wandel von Erziehungsstilen in der Unterschicht liegen meines Wissens zwar keine Untersuchungen vor, aber ich vermute, dass die sozial bedingten Unterschiede in den schulischen Leistungen seit den 1970er Jahren größer geworden sind (Steinig u.a. 
2009), weil sich zum einen in Mittelschichtfamilien die Erziehungsbemühungen mit dem Wandel vom permissiven zum autoritativen Erziehungsstil intensiviert haben, zum andern aber in Unterschichtfamilien der autoritäre Erziehungsstil tendenziell weniger praktiziert wurde, der vernachlässigende hingegen zunahm. Autoritär erziehende Eltern setzen Normen und Werte, nach denen sich Kinder zu richten haben, unterbinden Diskussionen und versuchen, mit Befehlen und Strafen ihren Willen durchzusetzen. Schulbezogene Fragen werden zwar nicht mit den Kindern besprochen, aber die Ansprüche der Schule, etwa in Bezug auf die Erledigung der Hausaufgaben, werden formal unterstützt. Eltern mit einem vernachlässigenden Erziehungsstil haben es hingegen aufgegeben, schulische Ansprüche zu unterstützen. Es interessiert sie nicht, welche Hausaufgaben zu erledigen sind, was in der Schule gelesen wird oder welche Prüfungsarbeiten anstehen, während sich HelikopterEltern engagiert darum kümmern.

Die wachsende soziale Ungleichheit führt zu größeren Unterschieden in der Erziehung. Während in der Mittelschicht mehr in Erziehung investiert wird, lassen die erzieherischen Bemühungen in der Unterschicht nach, da sich ihre Investition offenbar weniger rentiert, denn es wird zunehmend schwieriger, sozial aufzusteigen; die Grenzen zwischen den sozialen Schichten werden dichter und die soziale Segregation hat zugenommen (Friedrichs/Triemer 2008). Grundschullehrkräfte sind heute weitgehend davon überzeugt, dass ein gemeinsamer Unterricht für alle Schüler kaum noch durchführbar sei. Bereits beim Schuleintritt zeigen sich enorme Unterschiede, vor allem in den sprachlichen Kompetenzen der Kinder, die sich spätestens nach dem Übertritt in die unterschiedlichen Sekundarschulen noch einmal deutlich verstärken. Dieser Schereneffekt entsteht in unserem dreigliedrigen Schulsystem nicht nur, weil auf dem Gymnasium quantitativ und qualitativ mehr gelernt wird als in Schularten mit geringerem Bildungsanspruch, sondern auch, weil sich die einzelnen Schulkulturen mit ihren jeweiligen Peer Groups sozial ausdifferenzieren und unterschiedliche lebensweltliche und sprachliche Gemeinschaften bilden, die sich in ihren drei wesentlichen kommunikativen Settings Familie, Unterricht und Peer Group stützen und verstärken.

Die Ausdifferenzierung des Sprachverhaltens und des damit verbundenen kulturellen Kapitals und des Habitus (Bourdieu 1987) beruhen auf einer milieuspezifischen familialen Sozialisation, die sich zum einen auf objektive soziale Parameter wir Beruf, Bildung und Einkommen zurückführen lassen, zum anderen auf subjektive Einstellungen, lebensweltliche Orientierungen und Interessen. Wie sich die unterschiedlichen habituellen und sprachlichen Verhaltensweisen in amerikanischen Familien aus der Unterschicht und der Mittelschicht im alltäglichen Miteinander von Eltern und Kindern entwickeln, hat Annette Lareau (2011) in einer ethnographischen Studie mit 12 Familien eindrücklich darstellen können. In Bezug auf die Erziehung in der Mittelschicht hat sie beobachtet:

[...] middle-class parents engage in a process of concertet cultivation. From this, a robust sense of entitlement takes root in the children. This sense of entitlement plays an especially important role in institutional settings, where middle-class children learn to question adults and address them as relative equals. (Lareau 2011, S. 2) 
Die Erziehung in der Arbeiterschicht hat eine andere Ausrichtung:

For them, the crucial responsibilities of parenthood do not lie in eliciting their children's feelings, opinions, and thoughts. Rather, they see a clear boundary between adults and children. Parents tend to use directives: they tell their children what to do rather than persuading them with reasoning. [...] Their parents and guardians facilitate the accomplishment of natural growth. [...] For working-class and poor families, the cultural logic of child rearing at home is out of synch with the standards or institutions. (Lareau 2011, S. 3)

Mittelschicht-Eltern betreiben nach Lareau concertet cultivation, also ein konzertiertes Erziehungsprogramm, mit dem ihre Kinder in die Lage versetzt werden, an der dominanten Kultur ihres Landes aktiv teilzunehmen. Sie entwickeln dabei eine Anspruchshaltung, dass ihnen die kulturellen Errungenschaften zustehen und sie von ihnen extensiv genutzt werden können: Nicht nur eine hochwertige schulische Bildung, auch außerschulische Bildungsangebote wie Theater-, Konzert- und Museumsbesuche sowie sportliche Aktivitäten, die neben dem Geist auch den Körper, das soziale Miteinander und Durchsetzungsvermögen fördern sollen. All diese Aktivitäten werden von den Eltern im kommunikativen Austausch mit ihren Kindern begleitet, unterstützt und kommentiert, sodass die Kinder die Ansprüche der Eltern als ihre eigenen Ansprüche annehmen und das Programm der Selbst-Kultivierung im Idealfall eigenständig und selbstbewusst vorantreiben.

In der Unterschicht (working class) ist Erziehung weniger ambitioniert. Hier ist man damit zufrieden, wenn Kinder >natürlich $<$ aufwachsen können. Im direkten Kontakt mit ihren Eltern werden sie zwar häufiger reglementiert und zurückgewiesen, aber wenn sie sich dem unmittelbaren Einfluss ihrer Eltern entziehen, können sie ihre Freizeit weitgehend frei nach ihren eigenen Vorstellungen und Wünschen gestalten. Die Schule ist aus ihrer Sicht eher ein notwendiges Übel, jedenfalls keine Institution, die als Königsweg für ein erfolgreiches Leben akzeptiert wird. Auch ihre Eltern sehen Schule als eine Institution, zu der man Distanz halten sollte, da sie Misstrauen erzeugt und mit Einschränkungen, Zwängen und Frustrationen assoziiert wird. Diese Distanz äußert sich etwa darin, dass die Eltern nicht den Kontakt zu den Lehrkräften ihrer Kinder suchen und Elternsprechtagen fernbleiben. Die sozialen Milieus zu denen Lehrkräfte gehören, unterscheiden sich von den Milieus der sozialen Unterschicht: nicht nur in ihren Vorstellungen von Erziehung und Lebensgestaltung, sondern nicht zuletzt auch in ihrem kommunikativen Verhalten.

Bildungssprache kann man in diesem subkulturell unterschiedlich geprägten Kontext nicht isoliert betrachten. Sie ist keine sprachliche Varietät, die man wie eine Fremdsprache in der Schule erlernen kann, sondern Ausdruck eines gebildeten Habitus, der sich tief in der Persönlichkeit eines Menschen verankert hat. In familialen Milieus der Mittelschicht wird der Habitus eines Doing gebildet gepflegt und zwar besonders in rituell geprägten Situationen, in denen ein Doing Family gestaltet wird, etwa beim gemeinsamen Abendessen und einer sich daran anschließenden GuteNacht-Geschichte: Situationen, die den Kindern eine familiale Identität geben, die über den Tag und die Schule hinaus den gesamten Lebensweg prägen. 


\section{Von familialer zu unterrichtlicher Kommunikation}

Bei einem ersten oberflächlichen Vergleich von familialer mit unterrichtlicher Kommunikation sollte man vermuten, dass die kommunikativen Anforderungen an Schüler im Unterricht deutlich höher sind als in der Familie. Ein Vergleich zwischen diesen beiden Settings, der im Rahmen der Bristol Study of Language Development at Home and at School (Wells 1982) durchgeführt wurde, kommt jedoch zu einem gänzlich anderen Ergebnis:

Compared with their experiences at home, children at school were found to play a much less active role in conversation. They initiated fewer interactions, asked fewer questions, and took fewer turns per interaction. Their utterances were syntactically simpler, contained a narrower range of semantic content, and less frequently referred outside the here and now. Indeed almost half their utterances were elliptical or moodless sentence fragments, often minimal responses to requests for display. [...] In contrast with their parents, these children's teachers dominated conversation, initiating the majority of interactions, predominantly through requests for display. They were also more than twice as likely to develop their own meanings as they were to extend those contributed by the children, this ration being almost the exact opposite of that found in the speech of the parents. [...] we found that the schools were not providing a linguistically rich environment able to provide compensation for those believed to be deprived at home. On the contrary, there were no homes that did not provide richer opportunities than the schools [...] for learning through talk with an adult. Clearly there is a mismatch between teachers' aims and the reality of classroom practice. (Wells \& Wells 1984, S. 193f.)

Diese Beobachtungen aus der Bristol-Studie, einer in den 1980er Jahren durchgeführten Langzeitstudie mit 128 Kindern vor ihrem Schuleintritt und danach, enttäuschen unsere Erwartungen an einen kommunikativ förderlichen Unterricht in eklatanter Weise. Das Ziel, Kinder von einem informellen Sprachduktus zu einem formellen, dekontextualisierten und konzeptionell schriftlichen $\mathrm{zu}$ führen, bei dem Schüler lernen, Sachverhalte zu erklären und Standpunkte eigenständig argumentativ zu vertreten, kann mit dieser Form der kommunikativen Interaktion offensichtlich nicht erreicht werden; dies wird vielmehr verhindert! Dass diese scharfe Kritik aus den 1980er Jahren immer noch aktuell ist, zeigen vielerorts neuere Beobachtungen, so auch an finnischen Schulen, deren Schüler in PISA-Testungen Spitzenwerte erreichen. Auch hier wird der Lehrstoff traditionell lehrerzentriert präsentiert; die Lehrkräfte beanspruchen die weitaus größte Redezeit für sich, während Schüler vorwiegend knappe Antworten geben (van Ackeren/Kühn 2010, S. 41 f.).

Für Kinder, die in bildungsnahen Familien mit einer anregungsreichen kommunikativen Kultur gelernt haben, sich weitgehend sachgerecht und auf Augenhöhe mit Erwachsenen zu unterhalten, ist eine rigide durch die Lehrkraft gesteuerte Gesprächskultur im Unterricht weniger problematisch als für Kinder aus bildungsfernen Familien, die in der Schule so nicht die Möglichkeit bekommen, ihre bildungssprachlichen Defizite durch eine aktive Teilhabe am Unterrichtsdiskurs auszugleichen. Es ist vielmehr zu erwarten, dass sich der kommunikative Graben im Laufe der Schul- 
zeit, vor allem nach dem Übergang auf die Sekundarstufe, noch vergrößert, denn die Wahrscheinlichkeit für Kinder mit familial bedingten bildungssprachlichen Defiziten, nicht auf ein Gymnasium wechseln zu können, ist in Deutschland besonders groß. Ist die Selektion beim Wechsel auf die verschiedenen Sekundarschularten mit ihren unterschiedlichen sprachbezogenen Ansprüchen erst einmal vollzogen, können kompensatorische Maßnahmen, etwa in einer Hauptschule, Realschule, Gesamtschule oder Stadteilschule, kaum noch greifen. Die unterschiedlichen kommunikativen schulischen Milieus entwickeln eine je spezifische Eigendynamik, die die schulische und damit auch die gesellschaftliche Selektion verstärkt. Es müsste also gelingen, bereits im Kindergarten, aber spätestens in der Grundschule die kommunikativen Verhältnisse so zu verändern, dass familial bedingte Defizite kompensiert werden können. Ich werde später dazu einen Vorschlag machen. Zuvor sollen noch weitere Unterschiede, aber auch Gemeinsamkeiten zwischen familialer und unterrichtlicher Kommunikation beleuchtet werden.

Die Art und Weise wie Mütter in einem qualitativ dichten sprachlichen Miteinander, wie etwa beim gemeinsamen Lesen von Bilderbüchern, Impulse geben und Fragen stellen, fördert die Entwicklung einer dekontextualisierten Sprachlichkeit. Wenn man von diesen bedeutsamen Sprachlernsituationen in der Mutter-KindKommunikation eine Verbindung zur Lehrer-Schüler-Kommunikation ziehen möchte und dabei von der Prämisse ausgeht, dass die Qualität der Impulse und Fragen einen entscheidenden Einfluss auf die sprachliche Entwicklung von Schülern hat, wird man feststellen müssen, dass sich die Klassifikation der Gesprächsthemen und die Rahmung des kommunikativen Settings (Bernstein 1990) grundsätzlich voneinander unterscheiden. Während die Mutter-Kind-Kommunikation privat gerahmt ist, herrscht im Klassenunterricht eine öffentliche, durch die Institution Schule geprägte Rahmung. Die Themen sind in der Mutter-Kommunikation offen; es gibt keine klassifizierenden Vorgaben, sich jeweils auf einen thematischen Fokus zu konzentrieren, wie dies im Unterricht der Fall ist.

Bei geringer Klassifikation und Rahmung in der Kommunikation zwischen Kindern und Erwachsenen ist es grundsätzlich leichter, von kontextualisierten zu dekontextualisierten Äußerungen zu wechseln und so für Kinder kognitiv und sprachlich nachvollziehbare Wege zu ermöglichen. Sind Gespräche hingegen stark klassifiziert und gerahmt, bleiben Sprecher in Bezug auf Inhalte, Intentionen und Gesprächsmodus eng begrenzt. Stark klassifizierte und gerahmte Gespräche kann man zum einen in sozial unterprivilegierten, bildungsfernen Familien finden, zum anderen im lehrerzentrierten Klassenunterricht. Kommunikation mit geringer Klassifikation und Rahmung lässt sich hingegen eher in Familien aus höheren sozialen Milieus finden und an Schulen mit offenen, schülerzentrierten Unterrichtskonzepten.

Wie eng oder weit Klassifikation und Rahmung in Gesprächen zwischen Kindern und Erwachsenen gegeben ist, hängt davon ab, wie viel davon Erwachsene zulassen. Entweder sie sind darauf bedacht, das durch Status, Rolle und Wissen gegebene asymmetrische Machtgefälle zwischen ihnen und den am Gespräch beteiligten Kindern durch eine weitgehende Kontrolle der Kommunikation aufrecht zu erhalten oder aber sie minimieren ihre Kontrolle, geben gewissermaßen Gedankenfreiheit, öffnen die Gesprächssituation, lassen zu, dass man, auch spielerisch und humorvoll, vom Hölzchen aufs Stöckchen kommt und sprachliche Brücken zwischen unterschiedli- 
chen Domänen und Modi baut. Selbstverständlich gibt es zwischen diesen beiden extremen Polen zahllose Übergänge, aber es ist für unsere Argumentation nützlich, die sprachlichen Möglichkeiten einmal von diesen beiden Polen her zu betrachten.

Wenn Klassifikation und Rahmung eng gehalten werden, führt das zu thematisch fokussierten Gesprächen, die auf einen sprachlichen Modus beschränkt bleiben: im familialen Setting auf einen informellen, alltagssprachlichen Modus, der sich an den kontextuellen Notwendigkeiten der unmittelbaren Lebenswelt orientiert; im unterrichtlichen Setting auf einen schulsprachlichen bzw. bildungssprachlichen Modus mit einer - je nach Klassenstufe - mehr oder weniger dekontextualisierten Sprachverwendung.

Das zentrale Motiv für ein eng klassifizierend und rahmend geführtes Gespräch liegt in dem Bemühen von Eltern wie Lehrkräften, Macht und Kontrolle in einer asymmetrischen Rollenkonstellationen aufrechtzuerhalten, wobei die Kinder sich den von den Erwachsenen gesetzten kommunikativen Bedingungen fügen sollen. Eltern, die diesen Gesprächsmodus favorisieren, praktizieren einen autoritären Erziehungsstil, der ihren Lebenserfahrungen als Arbeitnehmer in untergeordneten Positionen mit geringer Autonomie und wenig eigenständigen Handlungsoptionen entspricht. Im Gespräch mit Vorgesetzten ist es für sie am sinnvollsten, sich eng an deren inhaltliche und modale Vorgaben zu halten, also an die vorgegebene Klassifikation und Rahmung. Ein Ausbrechen aus diesem Muster könnte von Vorgesetzten als anmaßend oder ungehörig empfunden werden und zu einer Degradierung oder Entlassung führen. Eltern, in deren Familien sich diese Lebenserfahrung manifestiert hat, werden sie unbewusst in ihren Erziehungsstil einfließen lassen, da sie ihre Kinder so auf deren voraussichtlich eintretende berufliche Zukunft vorbereiten. Das ist aus ihrer Sicht rational und sinnvoll, da die Wahrscheinlichkeit für ihre Kinder, sozial aufzusteigen, um später in einer gehobenen Position die Gesprächsregeln setzen zu können, gering ist.

Hinzu kommt aber noch ein Umstand, der die physischen und kognitiven Voraussetzungen dieser Eltern betrifft. Auf die Gedankensprünge und die kreativen, manchmal auch schwer nachvollziehbaren Fragen ihrer Kinder steht ihnen schlicht keine adäquate Anschlusskommunikation zur Verfügung. Deren wissbegierige und neugierige Bemerkungen werden übergangen, weil sie sich überfordert fühlen, darauf einzugehen. Sie empfinden die Fragerei als nervig und unterbinden sie nicht selten mit brüsker Zurückweisung - nicht nur, weil ihnen die sprachlichen Mittel und das nötige Wissen fehlen, den von ihren Kindern begonnenen Gesprächsfaden weiterzuspinnen, sondern weil sie aufgrund ihrer Lebenssituation oft physisch zu erschöpft sind, um darauf einzugehen. Auch unbeholfene kindersprachliche ÄuBerungen mit ihren phonetischen, grammatischen und semantischen Unzulänglichkeiten, die oft ungewollt witzig geraten, werden von diesen Eltern eher selten als witzig empfunden, sondern als inkompetent und fehlerhaft, so dass es sich aus ihrer Sicht nicht lohnt, darauf einzugehen. Das autoritäre Erziehungsmuster kann so in ein vernachlässigendes (neglectful) Muster übergehen.

Man mag zunächst überrascht sein, dass eng klassifizierend und rahmend geführte Gespräche nicht nur in unterprivilegierten, bildungsfernen Familien vorherrschen, sondern ausgerechnet auch dort, wo Bildungswissen vermittelt werden soll, nämlich in einem vom Lehrer gesteuerten Klassenunterricht; hier allerdings nicht in 
einem alltagssprachlichen Modus der Nähe, sondern einem schul- bzw. bildungssprachlichen der Distanz. Die Setzung eines unterrichtlichen Gegenstandes wird von der Lehrkraft in der Regel widerspruchslos akzeptiert, wobei sie sich auf ihren staatlichen Auftrag, die Vorgaben des Bildungsplans zu erfüllen, berufen kann. Der Bildungsplan ist das maßgebliche Manual zur Klassifizierung der unterrichtlichen Themen und Ziele. Und das professionelle Selbstverständnis von Lehrkräften besteht darin, sich an diese Klassifikation zu halten und sie nicht durch Abweichungen zu verwässern. Wie sehr Lehrkräfte nach dieser Maßgabe handeln, wird mit der folgenden Begebenheit anschaulich.

Im ersten Schuljahr einer bayerischen Grundschule fiel ein Schüler der Lehrerin negativ auf, da er unvermutet, mitten im Sachunterricht, als es um ein gänzlich anderes Thema ging, der gesamten Klasse sein profundes Wissen über Giftschlangen mitzuteilen versuchte, von deren Gefährlichkeit er fasziniert war. Aber da sein Wissen nicht zum Unterrichtsthema passte, also zu einer anderen Klassifikation gehörte, sowie auch nicht durch eine von der Lehrerin erteilte Vergabe des Rederechts gerahmt war, sah sie sich gezwungen, diesen Schülerbeitrag als >nicht erwünscht $<$ zu unterbinden. Den Eltern dieses Erstklässlers teilte die Lehrerin mit, dass ihr Sohn wohl noch nicht >schulreif $<$ sei, da er die relativ rigiden Regeln zur Klassifikation und Rahmung des Unterrichts nicht beherrsche. Da im Elternhaus des Jungen ein permissiver Erziehungsstil vorherrschte, war er es gewohnt, jederzeit das sagen zu dürfen, was ihn gerade interessierte, und er konnte davon ausgehen, dass seine Eltern üblicherweise auf seine spontanen Einfälle eingehen und diese weiterspinnen.

Eine starke Klassifizierung im lehrerzentrierten Klassenunterricht geht normalerweise einher mit einer starken Rahmung durch die regulative Führung einer dominant agierenden Lehrkraft, die etwa zwei Drittel bis drei Viertel aller Äußerungen für sich beansprucht; das restliche Drittel verteilt sich auf alle Schülerinnen und Schüler, so dass im Durchschnitt auf einen Schüler nur wenige Sekunden kommen (Einsiedler 2000). Das typische unterrichtliche Äußerungsmuster beruht auf einem Dreischritt: auf eine Lehrerfrage erfolgt eine Schülerantwort, die wiederum von der Lehrkraft in einem bewertenden Sprechakt akzeptiert oder nicht akzeptiert wird. Wird sie nicht akzeptiert, werden weitere Schüler aufgerufen, bis schließlich ein Schüler eine Antwort abliefert, die die Lehrkraft zufriedenstellt. Auf die Äußerung eines Schülers geht normalerweise kein anderer Schüler unmittelbar ein; es findet kein Gesprächsaustausch unter den Schülern statt. Sie ergreifen auch höchst selten die Initiative und richten von sich aus Fragen zum Unterrichtsgegenstand an die Lehrkraft oder an die Mitschüler; sollte das dennoch geschehen, werden sie nur knapp von der Lehrkraft beantwortet oder übergangen, jedenfalls nicht dazu genutzt, inhaltlich genauer und ausführlicher darauf einzugehen und die übrigen Schüler in die Problematik, die hinter einer Schülerfrage steht, einzubeziehen. Die Lehrkraft setzt die Themen; den Schülern wird das nicht erlaubt. Schüler werden willkürlich vom Lehrer zur Beantwortung von Fragen aufgerufen, nicht nur diejenigen, die sich mit einem Handzeichen gemeldet haben. Sind Schülerarbeiten aus Lehrersicht unzureichend oder fehlerhaft, werden sie negativ sanktioniert, oft auch mit einer Zensur, ohne dass deutlich wird, nach welchen Kriterien die Bewertung erfolgt (Morais/ Neves 2001). 
Dieses unterrichtliche Setting bietet Schülern wenig Möglichkeiten, bildungssprachliche Kompetenzen zu entwickeln. In einem offenen, schülerzentrierten Unterricht mit schwacher Rahmung scheinen dagegen die Bedingungen, ein bildungsaffines Register zu entwickeln, deutlich günstiger zu sein. Nachteilig könnte in diesem Szenario aber sein, dass die Lehrkraft als Moderator und Lernbegleiter zu wenig als bildungssprachliches und rhetorisches Vorbild in Erscheinung tritt und stattdessen mit den Schülern in einem alltagssprachlichen, informellen, manchmal sogar jungendsprachlichen Modus kommuniziert. Auch dieses Szenario wäre nicht günstig für die Entwicklung einer elaborierten Sprachfähigkeit, die Bildungsprozesse unterstützt und mit der man sich im öffentlichen Raum Geltung verschaffen kann.

Der heutige Unterricht liegt zwischen diesen beiden Polen: Er ist in Deutschland weder extrem stark noch extrem schwach gerahmt, während die Klassifikation aufgrund der engen Orientierung an den Bildungsplänen normalerweise durchgehend stark bleibt, auch bei schwacher Rahmung. In Frankreich finden wir dagegen eine stärkere Rahmung als in Deutschland; in den skandinavischen Ländern ist sie schwächer als hierzulande. Allerdings gibt es zwischen den einzelnen Bundesländern deutliche Unterschiede. In den nördlichen und westlichen Bundesländern ist sie tendenziell schwächer, in den südlichen, insbesondere in Bayern, ist sie deutlich stärker und in den östlichen Bundesländern, besonders in Sachsen und Thüringen, scheint sie noch stärker als in Bayern zu sein (Steinig 2017).

\section{Literale Gesprächskultur im Unterricht}

Das lehrergeleitete Klassengespräch ist nach wie vor die am häufigsten praktizierte Sozialform bzw. Methode mit einem Anteil von knapp 50\% am Gesamtunterricht (Haag 2010, S. 167). Die seit den 1960er Jahren teilweise heftige Kritik an dieser Unterrichtsform, die Schülern wenig Möglichkeiten bietet, in einen bildungssprachlichen Diskurs mit der Lehrkraft oder den Mitschülern zu treten, hat bis heute zu keiner wesentlichen Änderung geführt. Nach wie vor werden Schüler dazu genötigt, auf eng geführte Lehrerfragen mit sprachlich anspruchslosen Kurzantworten ein kognitiv wenig anregendes Gespräch am Laufen zu halten. Dass sich unter diesen rigiden kommunikativen Bedingungen produktive bildungssprachliche Kompetenzen entwickeln können, ist nur schwer vorstellbar. Eine tendenzielle, wenn auch nicht grundlegende Verbesserung kann man erreichen, wenn die Qualität der Lehrerfragen gesteigert wird und Lehrpersonen auf die Schülerbeiträge anders eingehen.

Um den engführenden Dreischritt Lehrerfrage/-impuls - Schülerantwort - Lehrerrückmeldung hinter sich zu lassen, müssten Lehrkräfte versuchen, Schüler in kognitiv anregende und sprachlich anspruchsvolle Gespräche zu verwickeln. Dazu sollten sie ihre Schüler zu längeren Gesprächsbeiträgen auffordern und ihnen Zeit geben, umfassendere Beschreibungen und Erklärungen zu formulieren, damit die üblichen Lehrerfragen, die sich wohl nie ganz vermeiden lassen, in ihrer Häufigkeit zurückgedrängt werden könnten. Wichtig wären auch Anschlussfragen an Schüler, die eine kurze, unzureichende oder unverständliche Antwort gegeben haben. Damit würde Schülern deutlich, dass die Lehrkraft Sachverhalte vertiefend mit ihnen diskutieren möchte. Schließlich wäre es für ein ertragreiches Klassengespräch wichtig, 
einzelne Schülerbeiträge aufeinander zu beziehen und miteinander zu vernetzen, damit Schüler auch direkt untereinander ins Gespräch kommen, nicht nur über die Lehrkraft als Gesprächsleiter.

Einige wenige Forscher haben sich mit der Verbesserung von Lehrerückmeldungen beschäftigt, die dazu führen, dass sich Schüler ausführlicher äußern und Lerngegenstände tiefer durchdringen (vgl. Pauli 2010). Anstatt einer kurzen bewertenden Rückmeldung (evaluation act) ist es förderlicher, Schüleräußerungen so aufzugreifen, dass sich daran eine längere Anschlusskommunikation entwickeln kann. Durch dieses Revoicing fühlen sich Schüler nicht mehr als unbedeutende Stichwortgeber oder nützliche Lückenfüller für den von Lehrer gesteuerten Unterrichtsverlauf, sondern erfahren Wertschätzung, die sie motiviert, sich am Unterrichtsgespräch zu beteiligen. So lassen sich die schülerseitigen Gesprächsanteile vergrößern und ihre Qualität erhöhen.

Revoicing geht über die Evaluation (z. B. als zustimmende Wiederholung der Schüleräußerung) hinaus, macht die Beiträge für die Diskussion verfügbar, drückt eine grundsätzliche Wertschätzung der Äußerung als eigenständigen Beitrag aus und unterstreicht die Rolle der Lernenden als ernstzunehmende Gesprächspartnerinnen und -partner sowie als Urheberinnen und Urheber einer Idee. (Pauli 2010, S. 149)

Die Schülerzahlen im Klassengespräch sind allerdings zu hoch, um allen Schülern längere Gesprächsbeiträge zu ermöglichen. Auch neuere Studien zeigen, dass »eine substanzielle Beteiligung der Lernenden an der Wissenskonstruktion « eher gering ist (Pauli 2010, S. 151). Wollte man die kommunikativen Verhältnisse in familialen Tischgesprächen unter unterrichtlichen Bedingungen zumindest ein stückweit simulieren, wäre das nur im Rahmen von Gruppenunterricht möglich. Hier haben Schüler die Möglichkeit, sich umfänglich am Gespräch zu beteiligen. Und da sie unter sich sind, werden diese Gespräche oft lebhaft von allen Beteiligten geführt. Lehrkräften wird empfohlen, während dieser Phasen nicht zu intervenieren, denn wenn sie sich einmischen, erstirbt das Schülergespräch abrupt und mutiert zu einer Art >Mini-Klassenunterricht< (Nürnberger Projektgruppe 2009).

Wenn »bei manchen Themen«, wie Ortner (2009, S. 2234f., Hervorh. im Original) meint, »nur die Ausführlichkeit der Behandlung das bildungssprachlich Besondere « sei, dann wäre es aus didaktischer Sicht sinnvoll, mit Gruppenarbeitsphasen einen Raum zu schaffen, in dem Schüler ungeschützt sehr viel längere Diskussionsbeiträge liefern können. Wenn, wie er weiter schreibt, »die Beherrschung der Bildungssprache nicht vor allem in der Kenntnis seltener Wörter (Quisquilien) besteht, sondern im vertieften Wissen hinter allen gebrauchten Wörtern «, dann lässt sich dieses Wissen am besten aufbauen, wenn man in substantiellen Gesprächen mit wenigen Gesprächspartnern gemeinsam ein Thema auslotet und dabei - notwendigerweise und gleichsam nebenbei - Wörter und Wortgruppen benutzt, mit denen man einen Sachverhalt am besten ausdrücken kann. In der Terminologie der Funktionalen Linguistik M.A.K. Hallidays (2013) würde es in erster Linie nicht auf den Mode of discourse ankommen, also die formalen (bildungssprachlichen) Merkmale eines Textes wie Syntax oder Wortgebrauch, sondern das Field, den Inhalt und die kommunikative Absicht. 
Ein gut vorbereiteter Gruppenunterricht animiert Schüler zwar zu zahlreichen Beiträgen; sie bleiben aber vorwiegend auf einem informellen, teilweise jungendsprachlich geprägten Niveau. Ohne Beisein einer Lehrperson oder erwachsener Experten von außerhalb der Schule sehen sich die Schüler nicht genötigt, ihre Beiträge auf einem bildungssprachlich angemessenen Niveau zu formulieren. Ich möchte deshalb vorschlagen, Gruppenarbeitsphasen dazu zu nutzen, dass jeweils in einer Gruppe die Lehrkraft bzw. ein/e Experte/in am gesamten Gespräch teilnimmt und zwar in einer zurückhaltend moderierenden Weise, um möglichst ausführliche Schülerbeiträge zu ermöglichen.

Gespräche in Kleingruppen unter Beteiligung einer Lehrkraft würden sich stark von Gesprächen unterscheiden, in denen die Schüler unter sich bleiben. Der Tenor of discourse würde sich verändern, da sich die Machtverhältnisse von einer symmetrischen Gruppenkonstellation zu einer asymmetrischen verschieben. Das Machtgefälle zwischen Lehrkraft und Schülern bleibt in der Kleingruppe erhalten, aber die veränderte Gruppengröße hat einen fundamentalen Einfluss auf die Performanz der Äußerungen. In Kleingruppen bis zu einer Gruppengröße von etwa fünf Personen ist ein informelles Gesprächsverhalten funktional adäquat. Je größer eine Gruppe wird, desto schlechter sind informelle Gespräche möglich, da Äußerungen notwendigerweise formeller und elaborierter werden müssen. Ein Grund dafür sind die zunehmend geringer werdenden Möglichkeiten spontaner Interventionen der Zuhörer in Form von Rückfragen, Unterbrechungen oder Reformulierungen.

Diese beiden unterschiedlichen Settings - formeller Klassenunterricht in der Großgruppe und informelle Gruppenarbeit in der Kleingruppe - entsprechen auf pragmatischer Ebene der Unterscheidung zwischen der Sie- und der Du-Ebene. Für den Klassenunterricht, dem formellen Setting, verlangen die kulturellen Normen in Deutschland, zumindest ab der Sekundarstufe, dass die Lehrkraft gesiezt wird (vgl. Steinig 2017). Im informellen Gruppengespräch, auch unter Beteiligung einer Lehrkraft, würde der Tenor eigentlich die $D u$-Ebene verlangen, aber ein situativ bedingter Wechsel zwischen der $\mathrm{Du}$ - und Sie-Ebene widerspricht den kulturell gültigen Gesprächsregeln. Da die Lehrkraft während der Gruppenarbeit den Schülern nicht erlauben kann, geduzt zu werden, wird sie auch in dieser informellen Situation kaum so viel Nähe zwischen sich und den Schülern herstellen können, dass ein Gespräch entsteht, wie es in bildungsnahen Familien beim gemeinsamen Essen möglich ist. Würden Gespräche in Kleingruppen allerdings nicht mit der Lehrkraft, sondern mit (jüngeren) Experten von außerhalb der Schule stattfinden, wäre eine für alle gemeinsame $D u$-Ebene möglich. Derartige informell geführte Experten-Schüler-Gespräche hätten das größte Potential, sich interaktiv mit Wissen auseinanderzusetzen und dazu - ungesteuert - bildungssprachliche Kompetenzen zu entwickeln. Es ist nämlich nicht so, wie man in nahezu allen Veröffentlichungen zur Bildungssprache lesen kann, dass sie nur in einem konzeptionell schriftlichen Modus möglich wäre. Auf jeder wissenschaftlichen Tagung kann man beobachten, dass zwar in den Vorträgen und den sich daran anschließenden Diskussionen dezidiert konzeptionell schriftlich formuliert wird, aber in den Pausen zwischen den Veranstaltungen wird in informellen Kleingruppen konzeptionell mündlich geredet: spontan und ungeplant als Small Talk, changierend zwischen privatem und kollegialem Austausch, aber auch fachlichen Bezügen. 
Für Schüler und Studenten, die aus bildungsfernen Elternhäusern stammen, sind informell geführte, bildungsaffine Gespräche ungewohnt und weitgehend unbekannt. Sie gehen wohl, aus mangelnder Erfahrung, von der Annahme aus, dass Themen, die über allgemein bekanntes Wissen hinausgehen, in angemessener Weise nur auf einer konzeptionell schriftlichen Ebene zur Sprache gebracht werden können. Deshalb ist es eminent wichtig für sie zu erfahren, wie ungeschützt, unprofessionell, tentativ und alltagssprachlich man auch kognitiv anspruchsvolle Themen angehen kann.

Sie ermöglichen ihnen einen niederschwelligen Einstieg, an bildungssprachlichen Diskursen teilzunehmen. Wenn elaborierte und prestigeheischende Formulierungen im Modus konzeptioneller Schriftlichkeit durch informelle Direktheit ersetzt werden, in der Satzabbrüche, das Suchen nach einer adäquaten Wortwahl, Unterbrechungen und spontane Rückfragen möglich sind, gibt es weniger Hemmungen, sich daran zu beteiligen. Die bildungssprachliche Barriere fällt weg und man traut sich, auch als ein Sprecher, dem dieser Modus aus der Kommunikation mit den Eltern nicht vertraut ist, gewissermaßen als >Bildungssprachlerner $<$, sich an fachlich und politisch relevanten Gesprächen zu beteiligen. Ganz ähnlich wie beim Erlernen einer Fremdsprache wäre es weniger hilfreich, sich einen bildungssprachlichen Wortschatz ähnlich wie beim > Vokabellernen < einzuprägen, wie es Philipp \& Efing (2018) empfehlen. Eine Sprache erlernt man am effektivsten, wenn man sie aktiv in realen Situationen benutzt.

Im Internet findet man zahlreiche Beispiele für Gesprächssituationen, mit denen sich die These belegen lässt, dass der kognitive und fachliche Anspruch nicht darunter leiden muss, wenn Gespräche in einem informellen Modus auf der Du-Ebene geführt werden. Besonders instruktive Beispiele findet man in dem Youtube-Format Jung \& Naiv - Politik für Desinteressierte (www.jungundnaiv.de). Prinzipiell duzen sich in diesem Format die Gesprächspartner. Und falls der Interviewte einen nicht geläufigen Fachbegriff verwendet, wird er vom Interviewer gebeten, ihn zu ersetzen bzw. zu erläutern. In dem Interview, das Tilo Jung am 30.6.2019 mit Prof. Dr. Maja Göpel von Scientists for Future führt, sind die Eingangsfragen ausgesprochen direkt und informell:

\author{
Wer bist du? \\ Was machst du? \\ Wo bist du zur Schule gegangen? \\ Warst du ne gute Schülerin? \\ Hast du dich damals schon für Klimawandel interessiert? \\ (https://www.youtube.com/watch?v=3vhuFlVGBeI)
}

Die informellen Fragen entsprechen dem informellen Setting des Interviews: eine einfache Holzbank an einem roh gezimmerten unaufgeräumten Tisch in der Ecke eines Buchladens mit Kinderbüchern im Hintergrund, die legere Kleidung der Gesprächspartner, das einfache Aufnahmegerät. Man bekommt den Eindruck eines spontan arrangierten Treffens. Die Antworten von Maja Göpel sind jedoch keineswegs trivial, auch wenn sie informelle und jugendsprachliche Elemente enthalten (im Folgenden unterstrichen). 
Das macht mich so kirre, dass wir dabei die menschliche Art Technologie und soziales Miteinander so organisieren und anpassen müssen, dass sie nicht die Fähigkeit der Natur unterminieren, diese Grundlagen in Zukunft zur Verfügung zu stellen. Das ist ja genau die andere Weltanschauung, ne ....

Also ich finde diese Plakate, die wir draußen haben, total super. ... Das draußen ist ja so in your face bang. Ich sag dir, bewerb [sic] dich jetzt. Ich versuch das jetzt so doll in deine Alltagsstruktur einzubauen, dass du dem nicht entkommst.

Wenn man dieses oder andere Interviews aus Jung \& Naiv Schülern (ab etwa 14 Jahren) zeigt, können sie einen Eindruck davon bekommen, wie Erwachsene des öffentlichen Lebens mit einem hohen Maß an Bildungswissen (hier vor allem Wissenschaftler und Politiker) komplexe Zusammenhänge in einem informellen Duktus verständlich darstellen können.

Als Kontrast zu einem dieser informell geführten Interviews in Jung \& Naiv könnten Schüler der Sekundarstufe II ein Gespräch analysieren, das der Journalist Günter Gaus mit Hannah Arendt am 28. 10. 1964 im Rahmen des Fernsehformats Zur Person geführt hat. Nach einem Vorspann mit klassischer Musik beginnt Gaus so:

Frau Hannah Arendt, Sie sind die erste Frau, die in dieser Reihe porträtiert werden soll, die erste Frau, wenn auch freilich nach landläufiger Vorstellung höchst männlicher Beschäftigung: Sie sind Philosophin. Darf ich von dieser Vorbemerkung zu meiner ersten Frage kommen. Empfinden Sie Ihre Rolle im Kreise der Philosophen trotz der Anerkennung und des Respekts, den man Ihnen zollt, als eine Besonderheit. Oder berühren wir damit ein Emanzipationsproblem, das für Sie nie existiert hat?

Darauf antwortet Hannah Arendt:

$\mathrm{Ja}$, ich fürchte, ich muss erst einmal protestieren. Ich gehöre nicht in den Kreis der Philosophen. ${ }^{2}$

Als dieses Interview 2013, also 49 Jahre nach der Ausstrahlung im SWR, ins Netz gestellt wurde, führte das u. a. zu diesen Zuschauerkommentaren:

- beeindruckend wie sauber beide reden, ohne >ähm< oder andere fehler [sic]

- Herr Gaus formuliert seine Sätze so wunderbar.

- Ich bin begeistert: Was für eine spannende, kenntnisreiche Debatte, geführt in einer Sprache, die heute wohl als druckreif gelten dürfte ... und der selbst nach heutigem Standard die meisten gebildeten Menschen nicht mächtig sind.

Es ist bezeichnend und erstaunlich zugleich, wie sehr sich manche Menschen von einem gehobenen bildungssprachlichen Duktus beeindrucken lassen. Wenn man diesen Kommentatoren zum Vergleich das Interview mit Maja Göpel vorlegen würde, kämen sie vermutlich zu dem Ergebnis, dass es in den 55 Jahren, die zwischen den beiden Interviews in den Formaten Jung \& Naiv und Zur Person liegen, zu einem sprachlichen Verfall gekommen sei.

\footnotetext{
2 https://www.youtube.com/watch?v=J9SyTEUi6Kw (gesehen am 28.11.2019).
} 
Beim Vergleich der beiden Interviews, den man in der Sekundarstufe II oder mit Studierenden durchführen könnte, müsste deutlich werden, dass das Gespräch mit Maja Göpel inhaltlich keineswegs oberflächlicher ist als das mit Hannah Arendt, obwohl es wesentlich informeller geführt wurde. Es besteht allenfalls eine indirekte Beziehung zwischen dem Grad konzeptioneller Schriftlichkeit und der kognitiven Durchdringung eines Sachverhalts.

Fließende Übergänge von Bereichen mit geringem und hohem kognitivem und sprachlichem Anspruch können als transitorische Felder von Lernern genutzt werden, um in eine Zone der nächsten Entwicklung zu gelangen (Wygotski 1987, S. 83). Auf rezeptiver Ebene wären das beispielsweise Interviews im Jung \& Naiv-Format, um zu erkennen, wie kognitiv anspruchsvolle Gespräche auf einer weitgehend informellen Ebene möglich sind. Auf produktiver Ebene könnten das Kleingruppengespräche im Rahmen des Gruppenunterrichts sein, an denen eine Lehrkraft oder ein Experte beteiligt wären - hier auch, um Unterschiede zwischen einer informelleren $D u$-Ebene und einer formelleren Sie-Ebene in fachbezogenen Gesprächen zu erfahren und sich dabei die dazu notwendigen sprachlichen Mittel in der Gesprächspraxis anzueignen.

Methodisch könnte man dabei im Gruppenunterricht so verfahren, dass die Schülerinnen und Schüler, die in ihrer Kleingruppe mit einer Lehrkraft oder einem Experten fachliche Erklärungen und Argumente erkannt, ausgetauscht und eingeübt haben, im zweiten Schritt jeweils einzeln in die übrigen Kleingruppen gehen, um dort ihr Wissen mit ihrer neu gewonnenen kommunikativen Expertise anzuwenden und so niederschwellig weiterzugeben. Diese Methodik beruht auf dem Tutor-TuteePrinzip, bei dem der Tutor stärker profitiert als der Tutee, da er den aktiveren Part übernimmt. Dabei muss gewährleistet werden, dass alle Schülerinnen und Schüler die Tutoren-Rolle einnehmen können, insbesondere und verstärkt aber diejenigen, die in ihren bildungsfernen Elternhäusern diese kommunikativen Erfahrungen beim Erklären und Argumentieren nicht machen konnten.

Dieser transitorische Erfahrungs- und Übungsraum darf nicht auf der kommunikativen Ebene beschränkt bleiben; also nicht losgelöst von inhaltlichen Feldern, den Fields of discourse, praktiziert werden, denn sonst wäre es ein Üben um des Übens willen mit geringer Nachhaltigkeit. Inhaltliche Felder, die geeignet wären, um transitorische Erfahrungen beim Verstehen komplexer Zusammenhänge zu ermöglichen, lassen sich grundsächlich in allen fachlichen, wissenschaftlichen und politischen Bereichen finden. Ich möchte hier einen Bereich herausgreifen, mit dem anschaulich gezeigt werden kann, wie ein kognitiver Transfer vom Hier und Jetzt unmittelbarer Erfahrung zu hypothetischen Modellen einer näheren und ferneren Zukunft möglich wäre, nämlich das Thema Wetter und Klima.

Das Wetter ist Menschen so nahe und konkret wie kaum ein anderer Lebensbereich. Darüber wird häufig gesprochen, besonders in unseren Breiten, wo das Wetter relativ häufig wechselt. In Kontaktgesprächen oder als Einstieg zu längeren Gesprächen wird meist kurz darauf hingewiesen: auf das Wetter in der unmittelbaren Gegenwart, auf das noch vor kurzer Zeit herrschende Wetter und das in den nächsten Stunden und oder Tagen zu erwartende Wetter. Um darüber zu sprechen, sind keine größeren kognitiven Anstrengungen nötig; die sprachlichen Mittel, um sich auf das Wettergeschehen zu beziehen, stehen allen Sprechern einer Sprachgemeinschaft 
zur Verfügung. Es sind meist hochgradig erwartbare Äußerungen, Kollokationen und idiomatische Wendungen. Wer eine Fremd- oder Zweitsprache lernt, wird sich bereits ganz zu Beginn des Spracherwerbs damit vertraut machen: Schönes Wetter heute. Hoffentlich bleibt es noch ein wenig so schön. Man weiß gar nicht, was man anziehen soll. Regen war auch mal dringend nötig. Diese Nacht hat es gefroren, usw.

Über das Klima zu sprechen, ist dagegen kognitiv wie auch sprachlich wesentlich anspruchsvoller. Es lässt sich nicht konkret im Hier und Jetzt erfahren, sondern nur anhand unzähliger Wetterdaten über einen mindesten zwanzigjährigen Zeitraum extrapolieren. Das globale Klimageschehen wie das Klima in einzelnen Regionen der Erde wird primär von Klimatologen in wissenschaftlichen Darstellungen in einer hochgradig abstrakten Sprache mit grafisch aufbereiteten Daten veröffentlicht. Von diesen wissenschaftlichen Texten ausgehend findet man populärwissenschaftliche und journalistische Texte sowie Texte von Laien in Internetforen, also eine sprachlich vielschichtige Form der Darstellung, an der sich seit jüngster Zeit im Rahmen der Bewegung Fridays for Future immer mehr Jugendliche beteiligen. Wir haben es hier also mit einem transitorischen Feld zu tun, das enorme Chancen bietet, Schülerinnen und Schülern Zugänge zu einem bildungssprachlichen Diskurs zu verschaffen.

Die bildungssprachliche Barriere, die mit der Bewegung Fridays for Future sichtbar wird, zeigt sich nun aber darin, dass zu dieser Bewegung nahezu ausschließlich Gymnasiasten gehören. ${ }^{3}$ Das liegt zum einen daran, dass sich Schülerinnen und Schüler anderer Schularten nicht an den gymnasial dominierten Foren beteiligen, da sie Sorge haben, bildungssprachlich nicht mithalten zu können und sich keine sprachliche Blöße geben möchten. Zum anderen scheinen sich Schüler und Schülerinnen aus anderen Schularten eher auf das Hier und Jetzt ihrer Lebenswelt zu fokussieren, vor allem auf die unmittelbare Befriedigung von Konsumwünschen. Um sich aktiv gegen den Anstieg der Erderwärmung zu positionieren, müsste man einen Belohnungsaufschub in Kauf nehmen und Einschränkungen im Konsumverhalten akzeptieren, da dieses Engagement, wenn überhaupt, erst in einer ferneren Zukunft Früchte tragen wird. Doch dieses Muster einer zeitlich verzögerten Gratifikation (Deferred Gratification Pattern) entspricht einem bildungsbürgerlichen Erziehungskonzept; in der familialen Sozialisation bildungsferner Schichten bleibt der Zugang zu diesem Muster weitgehend verschlossen.

Wenn man ein Gegensatzpaar Gegenwart/Wetter und Zukunft/Klima konstruieren möchte, koinzidiert das mit sozial bedingten Unterschieden in der familialen Erziehung, die sich wiederum in den Schularten und ihren bildungssprachlichen Möglichkeiten spiegeln. Um die Verschiebung vom Hier und Jetzt der Gegenwart auf eine mögliche Zukunft sprachlich zu bewältigen, sind anspruchsvollere sprachliche Mittel erforderlich: konjunktivische und futurische Formen sowie mehr oder weniger abstrakte Formulierungen zur Beschreibung zukünftiger Szenarien und Modelle, während Formulierungen, die sich auf das Wetter beziehen, mit einfacheren und voraussagbareren Mustern realisiert werden können.

Am Beispiel des Klimadiskurses lässt sich erkennen, wie sehr die bildungssprachliche Oberfläche eines Textes oder eines Diskurses mit der sozialen Basis einer Ge-

\footnotetext{
${ }^{3}$ https://protestinstitut.eu/projekte/demonstrationsbefragungen/befragung-fridays-for-future/ (gesehen
} am 28.11.2019). 
sellschaft, ihren Milieus und Erziehungspraktiken verwoben ist. Wenn man einen didaktischen Ansatz finden möchte, die sozial bedingten, sprachlichen Divergenzen ein wenig zu verringern, sollte man transitorische Situationen mit kommunikativen Verhältnissen konfigurieren, die Kindern und Jugendlichen aus bildungsfernen Familien einen gangbaren Zugang ermöglichen, um sprachliches und fachliches Lernen miteinander zu verbinden und sich an öffentlichen Diskursen, die die Zukunft unserer Gesellschaft betreffen, kompetent, versiert und engagiert beteiligen zu können.

Funding Open Access funding provided by Projekt DEAL.

Open Access Dieser Artikel wird unter der Creative Commons Namensnennung 4.0 International Lizenz veröffentlicht, welche die Nutzung, Vervielfältigung, Bearbeitung, Verbreitung und Wiedergabe in jeglichem Medium und Format erlaubt, sofern Sie den/die ursprünglichen Autor(en) und die Quelle ordnungsgemäß nennen, einen Link zur Creative Commons Lizenz beifügen und angeben, ob Änderungen vorgenommen wurden.

Die in diesem Artikel enthaltenen Bilder und sonstiges Drittmaterial unterliegen ebenfalls der genannten Creative Commons Lizenz, sofern sich aus der Abbildungslegende nichts anderes ergibt. Sofern das betreffende Material nicht unter der genannten Creative Commons Lizenz steht und die betreffende Handlung nicht nach gesetzlichen Vorschriften erlaubt ist, ist für die oben aufgeführten Weiterverwendungen des Materials die Einwilligung des jeweiligen Rechteinhabers einzuholen.

Weitere Details zur Lizenz entnehmen Sie bitte der Lizenzinformation auf http://creativecommons.org/ licenses/by/4.0/deed.de.

\section{Literatur}

Ackeren, Isabell van/Kühn, Svenja Mareike (2010): Zwischen Anspruch und Realität ... Die Diskussion um Klassenführung und schülerorientierten Unterricht in Kanada und Finnland. In: Thorsten Bohl u. a. (Hg.): Selbstbestimmung und Classroom-Management. Bad Heilbrunn, S. 31-47.

Bernstein, Basil (1971): Class, Codes and Control. Vol. 1 - Theoretical Studies: Towards A Sociology of Language. London/New York.

Bernstein, Basil (1990): The structuring of pedagogic discourse. (= Class, codes \& control, Volume IV). London.

Bourdieu, Pierre (1987): Die feinen Unterschiede. Kritik der gesellschaftlichen Urteilskraft. Frankfurt.

Doepke, Matthias/Zilibotti, Fabrizio (2019): Love, money \& parenting. How economics explains the way we raise our kids. Princeton/Oxford.

Einsiedler, Wolfgang (2000): Von Erziehungs- und Unterrichtsstilen zur Unterrichtsqualität. In: Martin K.W. Schweer (Hg.): Lehrer-Schüler-Interaktion. Opladen, S. 109-128.

Entwisle, Doris/Alexander, Karl/Olson, Linda (2000): Summer Learning and Home Environment. In: R. Kahlenberg (Hg.): A Notion at Risk: Preserving Public Education as an Engine for Social Mobility. New York, S. 9-30.

Friedrichs, Jürgen/Triemer, Sascha (2008): Gespaltene Städte? Soziale und ethnische Segregation in deutschen Großstädten. Wiesbaden.

Graham, Ashley/McNamara, John K./Van Lankveld, Jackie (2011): Closing the Summer Learning Gap for Vulnerable Learners: An Exploratory Study of a Summer Literacy Programme for Kindergarten Children At-Risk for Reading Difficulties. In: Early Child Development and Care, 181(5), S. 575-585.

Haag, Ludwig (2010): Zu viel oder zu wenig Freiraum? Befunde zum guten Gruppenunterricht. In: Thorsten Bohl u. a. (Hg.): Selbstbestimmung und Classroom-Management. Bad Heilbrunn, S. 163-178.

Halliday, M. A. K. ( $\left.{ }^{4} 2013\right)$ : Halliday's Introduction to Functional Grammar. London.

Hart, Betty/Risley, Todd R. (1995): Meaningful differences in the everyday experience of young American children. Baltimore u. a.

Hart, Betty/Risley, Todd R. $\left({ }^{4} 2007\right)$ : Learning to talk. Baltimore/London/Sidney: Brookes.

Heller, Vivien (2012): Kommunikative Erfahrungen von Kindern in Familie und Unterricht. Passungen und Divergenzen. Tübingen.

Lakoff, George (1987): Women, fire, and dangerous things. What categories reveal about the mind. Chicago/London. 
Lange, Andreas/Xyländer, Margret (Hg.) (2011): Bildungswelt Familie. Theoretische Rahmung, empirische Befunde und disziplinäre Perspektiven. Weinheim/München.

Lareau, Annette (2011): Unequal childhoods. Class, race, and family live. Berkeley, Los Angeles, London.

Maccoby, Eleanor E./Martin, John A. $\left({ }^{4} 1983\right)$ : Socialization in the context of the family: Parent-child interaction. In: E. Mavis Hetherington (Hg.): Socialization, personality, and social development. Handbook of child psychology, Vol. IV, S. 1-101.

Morais, Ana/Neves, Isabel (2001): Pedagogic social contexts. Studies for a sociology of learning. In: Ana Morais u. a. (Hg.): Towards a sociology of pedagogy. New York u. a., S. 185-221.

Morek, Miriam (2012): Kinder erklären: Interaktion in Familie und Unterricht im Vergleich. Tübingen.

Nürnberger Projektgruppe (2009): Erfolgreicher Gruppenunterricht. Stuttgart.

Ortner, Hanspeter (2009): Rhetorisch-stilistische Eigenschaften der Bildungssprache. In: Ulla Fix/Andreas Gardt/Joachim Knape (Hg.): Rhetorik und Stilistik, Bd. 2. Berlin/New York, S. 2227-2240.

Pauli, Christine (2010): Klassengespräche - Engführung des Denkens oder gemeinsame Wissenskonstruktion selbstbestimmt lernender Schülerinnen und Schüler. In: Thorsten Bohl u. a. (Hg.): Selbstbestimmung und Classroom-Management. Bad Heilbrunn, S. 145-162.

Philipp, Maik/Efing, Christian (2018): Förderung von Sprache und Schriftsprache im Sekundarbereich. In: Cora Titz u.a. (Hg.): Konzepte zur Sprach- und Schriftsprachförderung entwickeln. Stuttgart, S. 198-213.

Steinhoff, Torsten (2019): Konzeptualisierung bildungssprachlicher Kompetenzen. Anregungen aus der pragmatischen und funktionalen Linguistik und Sprachdidaktik. In: Zeitschrift für Angewandte Linguistik 71, S. 327-352.

Steinig, Wolfgang u. a. (2009): Schreiben von Kindern im diachronen Vergleich. Münster u. a.

Steinig, Wolfgang (2017): Grundschulkulturen. Pädagogik - Didaktik-Politik. Berlin.

Steinig, Wolfgang/Huneke, Hans-Werner $\left({ }^{5} 2015\right)$ : Sprachdidaktik Deutsch. Berlin.

Weininger, Elliot B./Lareau, Annette (2009): Paradoxical pathways: An ethnographic extension of Kohn's findings on class and childrearing. In: Journal of Marriage and Family 71, S. 680-695.

Wells, Gordon (1982): The Bristol Study of language and development at home and at school. In: Early Years 2(2), S. 41-47.

Wells, Gordon/Wells, Jan (1984): Learning to talk and talking to learn. In: Theory into practice 23(3), S. 190-197.

Williams, Geoff (2001): Literacy pedagogy prior to schooling. Relations between social positioning and semantic variation. In: Ana Morais u.a. (Hg.): Towards a sociology of pedagogy. New York u.a., S. $17-45$.

Wygotski, Lew S. (1987): Ausgewählte Schriften. Bd 2: Arbeiten zur psychischen Entwicklung der Persönlichkeit. Köln.

Xyländer, Margret (2014): Die Familie als Bildungsgemeinschaft. Abendrituale in rekonstruktiver Analyse. Opladen/Berlin/Toronto. 